\title{
PLOS $\mid$ submission
}

S1 Table Schizophrenia association chi-squared general linear model coefficients for GTEx brain and GTEx/CommonMind consensus brain eQTLs compared to non-CNS eQTLs. The test statistics refer to a general linear model of all brain and non-CNS variants in the $\sim 9$ million variant template. The $\pi_{1}$ are therefore overestimated and do not represent genome-wide values.

\begin{tabular}{|c|c|c|c|c|c|c|c|}
\hline eQTL (n) & & $\bar{\beta}$ & $\beta$ (low 95\%) & $\beta$ (high 95\%) & $p$ & $\pi_{1}$ & $p_{\mathrm{MW}}$ \\
\hline \multirow{2}{*}{ Amygdala } & GTEx (1046) & -0.059 & -0.18 & 0.056 & 0.37 & 0.26 & 0.83 \\
\hline & GTEx\&CM (591) & -0.048 & -0.20 & 0.10 & 0.57 & 0.20 & 0.56 \\
\hline \multirow{2}{*}{ Cingulate } & GTEx (1976) & -0.039 & -0.12 & 0.046 & 0.42 & 0.38 & 0.68 \\
\hline & GTEx\&CM (1055) & -0.025 & -0.14 & 0.088 & 0.70 & 0.37 & 0.14 \\
\hline \multirow{2}{*}{ Caudate } & GTEx (2861) & -0.056 & -0.13 & 0.015 & 0.16 & 0.23 & 0.96 \\
\hline & GTEx\&CM (1280) & -0.018 & -0.12 & 0.085 & 0.76 & 0.30 & 0.12 \\
\hline \multirow{2}{*}{ Cerebellum } & GTEx $(4848)$ & 0.028 & -0.028 & 0.083 & 0.38 & 0.26 & 0.27 \\
\hline & GTEx\&CM (1827) & 0.063 & -0.023 & 0.15 & 0.20 & 0.31 & 0.0017 \\
\hline \multirow{2}{*}{ Cortex } & GTEx (3162) & -0.059 & -0.13 & 0.009 & 0.13 & 0.33 & 0.92 \\
\hline & GTEx\&CM (1620) & -0.024 & -0.12 & 0.067 & 0.64 & 0.37 & 0.13 \\
\hline \multirow{2}{*}{ Frontal Cortex } & GTEx (2454) & -0.026 & -0.10 & 0.05 & 0.55 & 0.30 & 0.93 \\
\hline & GTEx\&CM (1249) & 0.031 & -0.073 & 0.13 & 0.60 & 0.33 & 0.11 \\
\hline \multirow{2}{*}{ Hippocampus } & GTEx (1547) & 0.027 & -0.068 & 0.12 & 0.61 & 0.15 & 0.93 \\
\hline & GTEx\&CM (843) & 0.083 & -0.043 & 0.21 & 0.25 & 0.22 & 0.29 \\
\hline \multirow{2}{*}{ Hypothalamus } & GTEx (1492) & -0.04 & -0.14 & 0.057 & 0.47 & 0.24 & 0.95 \\
\hline & GTEx\&CM (814) & 0.036 & -0.092 & 0.16 & 0.62 & 0.21 & 0.77 \\
\hline \multirow{2}{*}{ Nucleus accumbens } & GTEx (2436) & -0.021 & -0.098 & 0.056 & 0.63 & 0.32 & 0.62 \\
\hline & GTEx\&CM (1155) & -0.026 & -0.13 & 0.082 & 0.68 & 0.32 & 0.098 \\
\hline \multirow{2}{*}{ Putamen } & GTEx (2022) & -0.045 & -0.13 & 0.038 & 0.34 & 0.27 & 0.89 \\
\hline & GTEx\&CM (993) & 0.0031 & -0.11 & 0.12 & 0.96 & 0.33 & 0.13 \\
\hline \multirow{2}{*}{ Substantia nigra } & GTEx (825) & -0.082 & -0.21 & 0.048 & 0.27 & 0.19 & 0.96 \\
\hline & GTEx\&CM (478) & -0.057 & -0.22 & 0.11 & 0.55 & 0.22 & 0.75 \\
\hline \multirow{2}{*}{ All } & GTEx (20659) & 0.013 & -0.0069 & 0.034 & 0.19 & 0.26 & 0.96 \\
\hline & GTEx\&CM (8992) & 0.094 & 0.067 & 0.12 & $7.73 \mathrm{E}-12$ & 0.30 & 0.00012 \\
\hline
\end{tabular}

